\title{
PRIMITIVE CONTRACTIONS OF CALABI-YAU THREEFOLDS I
}

\author{
GRZEGORZ KAPUSTKA AND MICHAŁ KAPUSTKA
}

\begin{abstract}
We construct examples of primitive contractions of Calabi-Yau threefolds with exceptional locus being $\mathbb{P}^{1} \times \mathbb{P}^{1}, \mathbb{P}^{2}$, and smooth del Pezzo surfaces of degrees $\leq 5$. We describe the images of these primitive contractions and find their smoothing families. In particular we give a method to compute the Hodge numbers of a generic fiber of the smoothing familly of each Calabi-Yau threefold with one isolated singularity obtained after a primitive contraction of type II. As an application we get examples of natural conifold transitions between some families of Calabi-Yau threefolds.
\end{abstract}

\section{InTRODUCTION}

A primitive Calabi-Yau contraction (cf. [Wi2]) is a birational morphism between Calabi-Yau varieties that does not factorize in the category of algebraic varieties. We say that a primitive contraction is of type II, if its exceptional locus is an irreducible surface that is mapped to a point. The dualizing sheaf of the exceptional surface is then antiample (i.e. the surface is a generalized del Pezzo surface). Moreover, from [G1] we know, that this exceptional locus is either a normal (Gorenstein) rational del Pezzo surface or a non-normal Gorenstein surface with $\omega_{E}^{-1}$ ample of degree 7 .

This paper grew up from an attempt to understand which del Pezzo surfaces do occur as the exceptional locus of a primitive contraction. We give examples of primitive contractions with exceptional locus being $\mathbb{P}^{2}, \mathbb{P}^{1} \times \mathbb{P}^{1}$ or a smooth del Pezzo surface of degree $\leq 5$. To obtain these examples we need to find a smooth Calabi-Yau threefold $X$ containing the chosen del Pezzo surface $D$ in such a way that each divisor on $X$ restricts to a multiple of the canonical divisor on $D$. Two types of constructions are given. In the first (construction 1 and 2) we consider the resolutions of double coverings of Fano varieties with index $\geq 2$ branched along the sum of two smooth surfaces $G^{\prime} \in\left|q K_{F}\right|$ and $\overline{D^{\prime}} \in$

The authors are scholars of the project which is co-financed from the European Social Fund and national budget in the frame of The Integrated Regional Operational Programme. 
$\left|(2-q) K_{F}\right|$, where $q$ is chosen such that $D^{\prime}$ is a smooth del Pezzo surface. The strict transform of $D^{\prime}$ will be an exceptional locus of a primitive contraction of type II. In this way we obtain primitive contractions of all considered del Pezzo surfaces.

Moreover, for del Pezzo surfaces of degree 3, 4, and 5 we find another construction (constructions 3 and 4). The Calabi-Yau threefold is then obtained as a small resolutions of a nodal threefold (a quintic and a complete intersection of two cubics, respectively) containing the anticanonical models of the considered del Pezzo surfaces.

The examples of primitive contractions of del Pezzo surfaces of degrees 6,7 , of $\mathbb{P}^{2}$ blown up in one point, and of singular del Pezzo surface are more difficult to find. This problem will be treated in $[\mathrm{K}$.

The second aim of this paper is to describe the smooth fibers of smoothing families of singular Calabi-Yau threefolds obtained by all constructions contained in this paper. Indeed the image of a primitive contraction of type II is a Calabi-Yau threefold with one isolated rational Gorenstein (i.e. canonical index 1), $\mathbb{Q}$-factorial singularity. By the results of Gross ([G1]) for most such Calabi-Yau threefolds there exists a flat family over the unit disc such that the generic fiber is smooth and the central fiber is this manifold. Such a family is called a smoothing of the singular Calabi-Yau threefold. We describe smooth fibers of these families (they are projective Calabi-Yau threefolds if the central threefold is projective).

In section 3 we describe the miniversal Kuranishi space of each singular Calabi-Yau threefold obtained as the image of a primitive contraction of type II. This gives us a method to compute the Hodge numbers of smoothings of these singular varieties.

We give moreover an exact description with equations in projective spaces of the singular Calabi-Yau threefolds considered in our constructions. To do this we first find the linear system that gives the primitive contraction and then describe the image of the morphism associated to this linear system.

In this paper we also study our examples in the context of conifold transition. The idea of conifold transitions goes back to Miles Reid (see [R1]) and was generalized in G2] in the following way. We say that the moduli spaces of two smooth Calabi-Yau 3-folds X and $\mathrm{Y}$ are joined by a conifold transition, if there exist a Calabi-Yau threefold $Z$ and two birational contractions $Z \rightarrow X^{\prime}$ and $Z \rightarrow Y^{\prime}$ such that $X^{\prime}$ (resp. $Y^{\prime}$ ) have a smoothing inside the moduli space of $X$ (resp. $Y$ ) (i.e. there exists a flat family $\mathcal{X} \rightarrow \Delta$ such that $\mathcal{X}_{0} \simeq X^{\prime}$ and $\mathcal{X}_{t} \in X$ for $t \neq 0)$. It is conjectured that all families of Calabi-Yau threefolds can 
be joined by a sequence of conifold transitions. The obtained results are summarized in the following table.

\begin{tabular}{|c|c|c|c|c|c|}
\hline $\operatorname{deg} D^{\prime}$ & $X^{\prime}$ & $\mathrm{C}=\operatorname{sing} \mathrm{X}^{\prime}$ & $\chi\left(\mathcal{Y}_{t}\right)$ & $Y^{\prime}$ & references \\
\hline 1 & $X^{\prime} \stackrel{2: 1}{\longrightarrow} F_{1}$ & $C_{3,1} \subset F_{1}$ & -204 & $Y_{6} \subset P(1,1,1,1,2)$ & thm. 4.2 \\
\hline 2 & $X^{\prime} \stackrel{2: 1}{\longrightarrow} F_{2}$ & $C_{3,1} \subset F_{2}$ & -156 & $Y_{3,4} \subset \mathbb{P}(1,1,1,1,1,2)$ & thm. 4.2 \\
\hline 3 & $X^{\prime} \stackrel{2: 1}{\longrightarrow} F_{3}$ & $C_{3,1} \subset F_{3}$ & -144 & $Y_{3,3} \subset \mathbb{P}^{5}$ & thm. 4.2 \\
\hline 4 & $X^{\prime} \stackrel{2: 1}{\longrightarrow} F_{4}$ & $C_{3,1} \subset F_{4}$ & -144 & $Y_{2,2,3} \subset \mathbb{P}^{6}$ & thm. 4.2 \\
\hline 5 & $X^{\prime} \stackrel{2: 1}{\longrightarrow} F_{5}$ & $C_{3,1} \subset F_{5}$ & -150 & $Y_{3,1,1} \subset G(2,5)$ & thm. 4.2 \\
\hline 8 & $X^{\prime} \stackrel{2: 1}{\longrightarrow} \mathbb{P}^{3}$ & $C_{6,2} \subset \mathbb{P}^{3}$ & -204 & $Y_{6} \subset \mathbb{P}(1,1,1,1,2)$ & thm. 4.3 \\
\hline 9 & $X^{\prime} \stackrel{2: 1}{\longrightarrow} \mathbb{P}^{3}$ & $C_{7,1} \subset \mathbb{P}^{3}$ & - & $\operatorname{deg}(63) \subset \mathbb{P}^{20}$ & thm. 4.3 \\
\hline 3 & $X^{\prime} \stackrel{2: 1}{\longrightarrow} \mathbb{P}^{3}$ & $C_{5,3} \subset \mathbb{P}^{3}$ & -200 & $Y_{5} \subset \mathbb{P}^{4}$ & thm. 4.3 \\
\hline 4 & $X^{\prime} \stackrel{2: 1}{\longrightarrow} Q_{2}$ & $C_{2,2,4} \subset \mathbb{P}^{4}$ & -176 & $Y_{2,4} \subset \mathbb{P}^{5}$ & thm. 4.4 \\
\hline 8 & $X^{\prime} \stackrel{2: 1}{\rightarrow} Q_{2}$ & $C_{1,2,5} \subset \mathbb{P}^{4}$ & -200 & $Y_{5}^{2} \subset \mathbb{P}^{14}$ & thm. 4.4 \\
\hline 4 & $X_{5}^{\prime} \subset \mathbb{P}^{4}$ & 36 ODP & -176 & $Y_{2,4} \subset \mathbb{P}^{5}$ & constr. 3 \\
\hline 5 & $X_{3,3}^{\prime} \subset \mathbb{P}^{5}$ & 28 ODP & -98 & $7 \times 7$ Pffafian $\subset \mathbb{P}^{6}$ & constr. 4 \\
\hline 3 & $X_{3,3}^{\prime} \subset \mathbb{P}^{5}$ & $12 \mathrm{ODP}$ & -144 & $Y_{2,2,3} \subset \mathbb{P}^{6}$ & thm. 3.14 \\
\hline 4 & $X_{3,3}^{\prime} \subset \mathbb{P}^{5}$ & $20 \mathrm{ODP}$ & -120 & $5 \times 5$ Pffafian $\subset \mathbb{P}^{6}$ & thm. 3.14 \\
\hline
\end{tabular}

TABLE 1.

Where $X_{d_{1}, d_{2}, .}$ denote a complete intersection of the indicated degrees in the indicated manifold and by $X_{5}^{2}$ we mean that $X$ is embedded by the double Veronese embedding. Moreover, $X \stackrel{2: 1}{\longrightarrow} F_{i}$ denotes an appropriate double cover of a Fano threefold with index 2, $Q_{2}$ a two dimensional quadric, and $n \times n$ Pffafian mean the variety defined by the $n-1 \times n-1$ Pffafians of an appropriated skew-symmetric matrix.

\section{ACKNOWLEDGEMENTS}

We would like to thank S. Cynk for his constant help. Some ideas from this paper grew up during our stay at the EAGER training site at Warwick University in spring 2004 we would like to express our gratitude to M. Reid for mathematical inspiration. We thank M. Gross, A. Langer, V. Nikulin, P. Pragacz, J. Wiśniewski for answering questions.

\section{Primitive Contractions}

By a Calabi-Yau threefold $X$ we mean a complex projective threefold with canonical singularities such that the canonical divisor $K_{X}=0$ and 
$h^{1}\left(\mathcal{O}_{X}\right)=0$. The aim of this paper is to study primitive contractions. Let us first recall some definitions from [Wi2].

\section{Definition 2.1.}

Let $X$ be a smooth Calabi-Yau threefold. We say that a birational morphism $\phi: X \rightarrow Y$ is a primitive contraction, if $Y$ is normal and one of the following equivalent conditions holds

a) $\operatorname{dim} \operatorname{Pic}_{\mathbb{R}}(Y)=\operatorname{dim} \operatorname{Pic}_{\mathbb{R}}(X)-1$;

b) $\phi$ does not factorize in the category of normal algebraic varieties.

\section{Definition 2.2.}

We say that a primitive contraction is:

- of type I if it contracts only finitely many curves,

- of type II if it contracts an irreducible surface down to a point,

- of type III if it contracts a surface down to a curve.

Our aim is to study type II contractions. First summarizing the results from G1] and Wi2 we obtain

\section{Theorem 2.1.}

The exceptional divisor of a primitive contraction of type II is a generalized del Pezzo surface (i.e. an irreducible Gorenstein surface with anti-ample dualizing sheaf). Moreover, the exceptional locus $E$ is either a rational Gorenstein del Pezzo surface, or a nonnormal surface with $\omega_{E}^{2}=7$ (that is not a cone), or a cone over an elliptic curve such that $\omega_{E}^{2} \leq 3$.

Proof. To prove that the exceptional locus is a generalized del Pezzo surface observe that $\pi: X \rightarrow Y$ is a resolution of singularities. Since $\pi$ is indecomposable, we conclude from [R3] that $\pi$ is a blow-up or an $\alpha$-blow-up of the singular point (to see this we can argue as in the proof of [R3, lemma 2.3]). Now from proposition 2.13 [R3] we conclude that the exceptional locus is a generalized del Pezzo surface. The second part follows from [G1, thm.5.2].

This paper grew-up from an attempt to understand the following problem.

\section{Problem 2.1.}

Which smooth del Pezzo surfaces can be contracted by a primitive contraction of type II.

To prove that a given contraction is primitive we will use the following characterization. 


\section{Theorem 2.2.}

$A$ del Pezzo surface $D$ can be contracted by a primitive contraction if and only if $D$ can be embedded in a smooth Calabi-Yau manifold $X$ in such a way that for each Cartier divisor $E \subset X$ we have $\left.E\right|_{D}=a K_{D}$, for some rational number a.

Proof. Let $H$ be an ample divisor on $X$. We claim that the $\mathbb{Q}$-divisor $a H+D$, where $a=\frac{\left(-K_{D}\right)^{2}}{H\left(-K_{D}\right)}$ (i.e. $\left.a H\right|_{D}=-K_{D}$ ), is big and nef. For this it is enough to observe that if $C$ is a curve contained in $D$ then $C .(a H+D)=0$. From the basepoint-free theorem we find an $n$ such that $|n(a H+D)|$ gives a birational morphism. To prove that we can find an integer $m$ such that the image of $\varphi_{|m n(a H+D)|}=: \varphi_{m}$ is a normal variety such that the morphism is an isomorphism outside from $D$ we

follow the proof of [Wi3, thm. 1.2]. Let $\varphi: X \stackrel{\gamma}{\rightarrow} Y \stackrel{\psi}{\rightarrow} Z$ be the Stein factorization such that $\gamma$ has connected fibers and $\psi$ is finite. The pull-back $\psi^{*}\left(H_{1}\right)=H$ of the hyperplane section of $Z$ is an ample divisor on $Y$ (see [Har, exercise $5.7 \mathrm{~d}]$ ). We choose an integer $r$ such that $r H=\psi^{*}\left(r H_{1}\right)$ is very ample. Since the Stein factorization of the morphism $\varphi_{r}$ factorizes through $\varphi_{1}$ the morphism $\varphi_{r}$ is birational and has normal image.

\section{Smoothings}

Most of the Calabi-Yau threefolds obtained after a primitive contraction of type II are smoothable.

Theorem 3.1 (cf. G1, Thm 5.8]).

Let $X \rightarrow Y$ be a primitive type II contraction with exceptional divisor $E$. Then $Y$ is smoothable unless $E \simeq \mathbb{P}^{2}$ or $E \simeq \mathbb{F}_{1}$.

We compute the Hodge numbers of the obtained smooth varieties.

\section{Theorem 3.2.}

Let $X \rightarrow Y$ be a primitive contraction of type II, such that $Y$ is smoothable. If $\mathcal{Y} \rightarrow \Delta$ is a 1-parameter smoothing, then

$$
h^{1,1}(X)-1=h^{1,1}\left(\mathcal{Y}_{t}\right) .
$$

Proof. (cf. [N, prop. 6.1]) Let $S$ be the universal Kuranishi space of $Y$ and $\mathcal{Z} \rightarrow S$ be the universal family. It follows from the Artin approximation theorem (see [A]) that we can find a flat projective morphism of algebraic varieties $\bar{\pi}: \overline{\mathcal{Z}} \rightarrow \bar{S}$ such that $S$ in an open subset of $\bar{S}$ and $\left.\bar{\pi}\right|_{S}=\pi$. From [KM, 12.1 .10 and 12.1.9] we can assume that $\overline{\mathcal{Z}}$ is $\mathbb{Q}$-factorial and that $\bar{\pi}$ has a section. This implies that every irreducible component of the relative Picard scheme $P i c_{\overline{\mathcal{Z}} \mid \bar{S}}$ is proper 
over $\bar{S}$ (see [K1, p.68] and [KM, 12.1.8]). Since $H^{1}\left(\mathcal{O}_{X}\right)=H^{2}\left(\mathcal{O}_{X}\right)=0$ from the general theory of Picard schemes (see [Kl, prop.5.19]) we obtain that each irreducible component of $P i c_{\overline{\mathcal{Z}} \mid \bar{S}}$ is étale over $\bar{S}$ (after shrinking $\bar{S}$ to a suitable Zariski open subset). We obtained that over $S$ the Picard scheme $P i c_{\bar{Y} \mid \bar{S}} \otimes_{\bar{S}} S=P i c_{Y \mid S}$ is a disjoint union of countably many copies of $S$. The natural structure group of $P i c_{Y \mid S}$ induces the group structure on the fibers, it follows that the rank of the Picard groups

$$
\rho\left(\mathcal{Y}_{t}\right)=\rho\left(\mathcal{Y}_{0}\right)=\rho(X)-1
$$

Remark 3.1. The above theorem follows also from [G2, prop 3.1] and [KM, 12.2.1.4.2], since $Y$ as the image of a primitive contraction of type II, has rational $\mathbb{Q}$-factorial singularities . Note that this theorem does not work for primitive contractions of type III. Indeed, since we cannot use [KM, 12.1.9], we cannot prove the properness of the irreducible components of $P i c_{\overline{\mathrm{Y}} \mid \bar{S}}$. See [G2, chapter 3] for an explicit example.

We first compute $h^{1,2}\left(\mathcal{Y}_{t}\right)-h^{1,2}(X)$ in the case when $Y$ has nonhypersurface singularities.

\section{Theorem 3.3.}

Let $\pi: X \rightarrow Y$ be a primitive contraction of type II, contracting a smooth del Pezzo surface of degree $5 \leq r \leq 8$. If $\mathcal{Y} \rightarrow \Delta$ is a 1 parameter smoothing, then for $t \neq 0$

(1) for $r=5, h^{1,2}\left(\mathcal{Y}_{t}\right)=h^{1,2}(X)+4$,

(2) for $r=6, h^{1,2}\left(\mathcal{Y}_{t}\right)=h^{1,2}(X)+1$ or $h^{1,2}\left(\mathcal{Y}_{t}\right)=h^{1,2}(X)+2$,

(3) for $r=7, h^{1,2}\left(\mathcal{Y}_{t}\right)=h^{1,2}(X)+1$,

(4) for $r=8, h^{1,2}\left(\mathcal{Y}_{t}\right)=h^{1,2}(X)+1$.

Proof. Recall that a miniversal Kuranishi space exists for compact complex spaces. For a Calabi-Yau threefold $X$ with canonical singularities this space is universal since $\operatorname{Hom}\left(\Omega_{X}^{1}, \mathcal{O}_{X}\right)=0$ (see [S2]). We denote by $\operatorname{Def}(X)$ the analytic germ of the Kuranishi space of $X$ or its suitable analytic representative. It follows from the openness of universality, (see [Bin, Satz 7.1(1)]) that the possible values of $h^{1,2}\left(\mathcal{Y}_{t}\right)$ will be equal to the dimensions of the irreducible components of $\operatorname{Def}(Y)$.

To compute these dimensions, we consider $T^{1}$ and $T_{l o c}^{1}$ the tangent spaces to $\operatorname{Def}(Y)$ and $\operatorname{Def}\left(Y^{\prime}, P\right)$ respectively, where $\operatorname{Def}\left(Y^{\prime}, P\right)$ denotes the miniversal deformation space of the germ $\left(Y^{\prime}, P\right)$ of the singularity obtained after the primitive contraction. From [S1, thm. 2] we have

$$
T^{1} \simeq H^{1}\left(Y-P, \Theta_{Y}\right) \simeq H^{1}\left(X-E, \Theta_{X}\right)
$$


and

$$
T_{l o c}^{1} \simeq H^{1}\left(Y^{\prime}-P, \Theta_{Y^{\prime}}\right) \simeq H^{1}\left(X^{\prime}-E, \Theta_{X^{\prime}}\right) .
$$

Here $\left(X^{\prime}, E\right) \rightarrow\left(Y^{\prime}, P\right)$ is the given minimal resolution.

We have the following exact local cohomology sequences

$$
\begin{gathered}
H^{1}\left(X^{\prime}, \Theta_{X^{\prime}}\right) \rightarrow H^{1}\left(X^{\prime}-E, \Theta_{X^{\prime}}\right) \stackrel{f^{\prime}}{\rightarrow} H_{E}^{2}\left(X^{\prime}, \Theta_{X^{\prime}}\right) \\
\uparrow \\
H^{1}\left(X, \Theta_{X}\right) \rightarrow H^{2}\left(X^{\prime}, \Theta_{X^{\prime}}\right) \\
\rightarrow H^{1}\left(X-E, \Theta_{X}\right) \stackrel{f}{\rightarrow} H_{E}^{2}\left(X, \Theta_{X}\right) \stackrel{\varphi}{\rightarrow} H^{2}\left(X, \Theta_{X}\right) .
\end{gathered}
$$

It follows from [G1, lem.4.5] that $\operatorname{im} f^{\prime}=\operatorname{im} f=: T^{\prime}$ and that the composition of maps $T^{1} \rightarrow T_{l o c}^{1} \rightarrow T^{\prime}$ is surjective.

We claim that $T^{1} \rightarrow T_{l o c}^{1}$ is in fact surjective. To see this, it is enough to prove that $f^{\prime}$ is an isomorphism onto its image. Recall from [Alt] and $[\mathrm{KL}]$ that $\operatorname{dim} T_{l o c}^{1}=9-r$. We shall prove that $\operatorname{dim} T^{\prime}=9-r$. Observe first, that

$$
\operatorname{dim} T^{\prime}=\operatorname{dim}(\operatorname{ker} \varphi)
$$

Following [G1, example 4.1] we dualize $\varphi$ and obtain the map

$$
H^{1}\left(X, \Omega_{X}^{1}\right) \rightarrow H^{0}\left(R^{1} \pi_{*}\left(\Omega_{X}^{1}\right)\right) .
$$

Since $\operatorname{deg} E \geq 5$ using the Theorem on Formal Functions and the exact sequence

$$
0 \rightarrow \Omega_{X}^{1} \otimes \mathcal{I}_{E}^{n-1} / \mathcal{I}_{E}^{n} \rightarrow \Omega_{X}^{1} \otimes \mathcal{O}_{X} / \mathcal{I}_{E}^{n} \rightarrow \Omega_{X}^{1} \otimes \mathcal{O}_{X} / \mathcal{I}_{E}^{n-1} \rightarrow 0
$$

we see that $H^{0}\left(R^{1} \pi_{*}\left(\Omega_{X}^{1}\right)\right) \simeq H^{1}\left(E, \Omega_{E}^{1}\right)$, and that $\varphi^{\vee}: H^{1}\left(X, \Omega_{X}^{1}\right) \rightarrow$ $H^{1}\left(E, \Omega_{E}^{1}\right)$ is the restriction morphism.

Since $H^{1}\left(X, \Omega_{X}^{1}\right) \simeq \operatorname{Pic}(X) \otimes \mathbb{C}$ and $\operatorname{dim}\left(H^{1}\left(E, \Omega_{E}^{1}\right)\right)=\operatorname{dim}(\operatorname{Pic}(E) \otimes$ $\mathbb{C})=10-r$ we deduce from thm. 2.2 that

$$
\operatorname{dim}\left(\operatorname{coker} \varphi^{\vee}\right)=9-r .
$$

The claim follows.

Following the discussion on page 211 in [G1] and using [G1, thm. 1.9, thm. 2.2] we see that

$$
\operatorname{Def}(Y)=\operatorname{Spec}(R / J)
$$

for $R=\Lambda\left[\left[y_{1}, \ldots y_{t}\right]\right]$, where $\operatorname{Spec}(\Lambda)$ is the base space of the miniversal deformation of the germ $\left(Y^{\prime}, P\right), t=\operatorname{dim}\left(\operatorname{ker}\left(T^{1} \rightarrow T_{l o c}^{1}\right)\right)$, and $J \subset$ $m_{\Lambda} R+m_{R}^{2}$ is some ideal (where $m_{R}=m_{\Lambda} R+\left(y_{1}, \ldots y_{t}\right)$ ).

Furthermore $m_{R} /\left(m_{R}^{2}+J\right)=m_{R} / m_{R}^{2}$ (this says that $T^{1}$ is isomorphic to the Zariski tangent space to $\operatorname{Spec}(R))$ and $\operatorname{Supp}(R / J)=\operatorname{Supp}(R)$.

From $\mathrm{Alt}$ and $[\mathrm{KL}$ ] we obtain a description of $\Lambda$.

- For $r=5$ we have $\Lambda=\mathbb{C}\left[\left[x_{1}, x_{2}, x_{3}, x_{4}\right]\right]$, 
- for $r=6$ we have $\Lambda=\mathbb{C}\left[\left[x_{1}, x_{2}, x_{3}\right]\right] /\left(x_{1} x_{3}, x_{2} x_{3}\right)$ then $\operatorname{Spec}(\Lambda)$ is a germ of a line and a plane meeting in one point,

- for $r=7$ we have $\Lambda=\mathbb{C}\left[\left[x_{1}, x_{2}\right]\right] /\left(x_{1}^{2}, x_{1} x_{2}\right)$ and $\operatorname{Spec}(\Lambda)$ is a line with a double origin,

- for $r=8$ we have two possibilities: if $E \simeq \mathbb{P}^{1} \times \mathbb{P}^{1}$, then $\Lambda=\mathbb{C}[[x]] ;$ if $E \simeq \mathbb{F}_{1}$, then $\Lambda=\mathbb{C}[[x]] /\left(x^{2}\right)$.

It follows that in our cases

$$
\operatorname{Def}(Y)=\operatorname{Spec}(R)
$$

To finish the proof, consider again the local cohomology sequence

$$
H_{E}^{1}\left(X, \Theta_{X}\right) \rightarrow H^{1}\left(X, \Theta_{X}\right) \rightarrow H^{1}\left(X-E, \Theta_{X}\right) \rightarrow T^{\prime}
$$

Since $\left(H_{E}^{1}\left(X, \Theta_{X}\right)\right)^{\vee} \simeq H^{0}\left(R^{2} \pi_{*}\left(\Omega_{X}^{1}\right)\right)=H^{2}\left(\Omega_{E}^{1}\right)=\left(H^{0}\left(\Omega_{E}^{1}\right)\right)^{\vee}=0$, we obtain that the map of germs $\operatorname{Def}(X) \hookrightarrow \operatorname{Def}(Y)$ is an embedding and its image has dimension $t=\operatorname{dim}\left(\operatorname{ker}\left(T^{1} \rightarrow T^{\prime}=T_{l o c}^{1}\right)\right.$ ) (we know that $\operatorname{Def}(X)$ is smooth). Moreover, the image of $\operatorname{Def}(X) \hookrightarrow$ $\operatorname{Def}(Y)$ is equal set theoretically to the principal fiber of $\operatorname{Spec}(R) \rightarrow$ $\operatorname{Spec}(\Lambda)$. It remains to compute the dimensions of the components of $\operatorname{Spec}\left(\Lambda\left[\left[y_{1}, \ldots y_{t}\right]\right]\right)$.

Remark 3.2. The above theorem where proved in the case $r=6$ in [N1] using different methods.

Remark 3.3. In fact we described the miniversal Kuranishi space of $Y$. We obtained that this space is the product of the miniversal space of the singularity that is the cone over the contracted del Pezzo surface with an appropriated germ of a linear space.

Remark 3.4. We compute $h^{1,2}\left(\mathcal{Y}_{t}\right)-h^{1,2}(X)$ in the case where $Y$ is obtained after a primitive contraction of smooth del Pezzo surface $E$ of degree $r \leq 4$ (note that in this case $Y$ has complete intersection singularities). In fact from thm. 3.2 it is enough to compute $\chi\left(\mathcal{Y}_{t}\right)-$ $\chi(X)$. Moreover, since we know that $\chi(Y)-\chi(X)=\chi(E)-1$ it is sufficient to find $\chi\left(\mathcal{Y}_{t}\right)-\chi(Y)$.

Using known facts about Milnor numbers we see that the last difference between the Euler characteristics $\chi\left(\mathcal{Y}_{t}\right)-\chi(Y)$ depends only on the Milnor number of the singularity of $Y$ so depends only on the degree of $E$. These differences will be computed using explicit examples (see Remark 4.1). We obtain the following

- if $r=4 h^{1,2}\left(\mathcal{Y}_{t}\right)=h^{1,2}(X)+7$,

- if $r=3 h^{1,2}\left(\mathcal{Y}_{t}\right)=h^{1,2}(X)+11$,

- if $r=2 h^{1,2}\left(\mathcal{Y}_{t}\right)=h^{1,2}(X)+17$,

- if $r=1 h^{1,2}\left(\mathcal{Y}_{t}\right)=h^{1,2}(X)+29$.

Compare this results with [MS]. 


\section{The Double Cover of a Fano threefold}

In this section we obtain Calabi-Yau threefold containing a given del Pezzo surface by resolving a double covering of a Fano threefold with index $\geq 2$ branched over a singular divisor. Recall that the index of a Fano manifold $F$ is the biggest number $g$ such that there exists an effective divisor $E$ with $-K_{F}=g E$.

In construction 1, 2, and 3 the covered Fano threefold has index 2, 3 , and 4 respectively. If the index is 3 the Fano threefold is isomorphic to a quadric in $\mathbb{P}^{4}$. If the index is 4 it is isomorphic to $\mathbb{P}^{3}$.

4.1. Construction 1. Let $F$ be a smooth Fano manifold with Picard number $\rho(F)=1$ and index 2. From [IP], $F$ is one of the following

\begin{tabular}{|c|c|c|}
\hline$H^{3}=r$ & $F$ & $\chi(F)$ \\
\hline \hline 1 & a generic hypersurface of degree 6 in $\mathbb{P}(1,1,1,2,3)$ & -38 \\
\hline 2 & a double cover of $\mathbb{P}^{3}$ branched along & \\
& a smooth surface of degree 4 & -16 \\
\hline 3 & a smooth cubic $X_{3} \subset \mathbb{P}^{4}$ & -6 \\
\hline 4 & a smooth intersection of two quadrics $X_{2,2} \subset \mathbb{P}^{s}$ & 0 \\
\hline 5 & $\begin{array}{c}\text { a linear section of the Grassmannian } G(2,5) \\
\text { in its Plücker embedding }\end{array}$ & 4 \\
\hline
\end{tabular}

TABLE 2.

In each of these cases $\left|-K_{F}\right|=|2 H|$, for some divisor $H$ on $F$. Moreover, if $r \geq 3$ the divisor $H$ is very ample, for $r=2$ it gives a 2:1 morphism and for $r=1$ it has exactly one base point (see [IP]). By the adjunction formula a general element $D^{\prime} \in|H|$ is a del Pezzo surface of degree $r$.

\section{Lemma 4.1.}

The normal double covering $\pi: X^{\prime} \rightarrow F$ branched along the sum of $D^{\prime}$ and a general $G^{\prime} \in|3 H|$ is a singular Calabi-Yau variety with a transversal $A_{1}$ singularity.

Proof. Observe that $D^{\prime}$ and $G^{\prime}$ meet transversally. This follows from the fact that the linear system $|3 H|$ is very ample. The singularity on the double cover is thus a transversal $A_{1}$ singularity along the strict transform $K$ of the intersection $D^{\prime} \cap G^{\prime}$ to $X^{\prime}$. This singularity is Gorenstein, so we compute 


$$
K_{X^{\prime}}=\pi^{*}\left(K_{F}\right)+\pi^{*}\left(\frac{D^{\prime}+G^{\prime}}{2}\right)=\pi^{*} K_{F}+\pi^{*}\left(-K_{F}\right)=0 .
$$

The blow up $\gamma: X \rightarrow X^{\prime}$ along the curve $K$ is a crepant resolution. Hence the threefold $X$ is a smooth Calabi-Yau threefold. Let $E$ be the exceptional divisor of $\gamma$. Denote by $D$ and $G$ the strict transforms of $D^{\prime}$ and $G^{\prime}$. Note that $D$ is isomorphic to $D^{\prime}$.

\section{Lemma 4.2 .}

The rank of the Picard group of $X$ is 2 (i.e. $\rho(X)=2$ ).

Proof. This follows from [C3, Example 1] because $\left|G^{\prime}-D^{\prime}\right|=|2 H|$ is ample.

Denote by $H^{*}$ the pull-back of $H$ to $X$. Observe that from the adjunction formula $\left.D\right|_{D}=K_{D}$ so $H^{*}+\left.D\right|_{D}=0$. We have the following

\section{Theorem 4.1.}

The linear system $|G|$ is base-point-free. Moreover,

- if $r \geq 3$, then $\varphi_{|G|}$ is a primitive contraction. Its image is a projectively normal threefold of degree $3 r$ in $\mathbb{P}^{r+2}$,

- if $r=2$ the morphism $\varphi_{|G|}$ is $2: 1$ onto a cubic in $\mathbb{P}^{4}$,

- if $r=1$ the morphism $\varphi_{|G|}$ is $3: 1$ onto $\mathbb{P}^{3}$.

Proof. Since $\pi^{*}(H) \simeq \pi^{*}\left(D^{\prime}\right)=D+E$ and $3 \pi^{*}(H) \simeq \pi^{*}\left(G^{\prime}\right)=G+E$ we obtain $G \in\left|H^{*}+D\right|=|E+3 D|$. From lemma 4.1 we conclude that $G \cap D=\emptyset$. If $r \geq 2$, then $\left.H^{*}\right|_{G}$ is base-point-free, hence $|G|$ is base-point-free. In the case $r=1$ the base point freeness follows from the fact, that a generic cubic in $\mathbb{P}(1,1,1,2,3)$ does not pass through the base point of the linear system $|H|$. Note that $\left.H^{*}\right|_{D}=-K_{D}$ and $\left.D\right|_{D}=K_{D}$ so $\left(H^{*}+D\right)^{3}=3 r$.

We compute $h^{0}\left(\mathcal{O}_{X}(G)\right)$ from the exact sequence

$$
0 \longrightarrow \mathcal{O}_{X} \longrightarrow \mathcal{O}_{X}(G) \longrightarrow N_{G \mid X} \longrightarrow 0 \text {. }
$$

Since $\mathcal{O}_{G}(H)=K_{G}$, we obtain

$$
h^{0}\left(\mathcal{O}_{X}(G)\right)=h^{0}\left(\mathcal{O}_{X}\right)+h^{0}\left(N_{G \mid X}\right)=1+h^{0}\left(\mathcal{O}_{G}(H)\right) .
$$

Now from

$$
O \longrightarrow \mathcal{O}_{F}(-2 H) \longrightarrow \mathcal{O}_{F}(H) \longrightarrow \mathcal{O}_{G}(H) \longrightarrow O
$$

we have $h^{0}\left(\mathcal{O}_{G}(H)\right)=h^{0}\left(\mathcal{O}_{F}(H)\right)$.

Assume that $r \geq 3$. The embeddings from table 1 are given by the linear system $|H|$, thus

$$
h^{0}\left(\mathcal{O}_{F}(H)\right)=r+2
$$


The morphism $\varphi_{\left|H^{*}+D\right|}$ is then generically $1: 1$ as it does not factorize through $\pi$. Observe moreover, that the morphism $\varphi_{\left|H^{*}+D\right|}$ does not contract any curve not contained in $D$ (a curve contained in $E$ cannot be contracted, since the elements of $\left.\left(D+\left|H^{*}\right|\right)\right|_{E}$ separate points from different fibers of $E \rightarrow K$ and $G \cap D=\emptyset)$.

To show that the image of $\varphi_{|G|}$ is projectively normal we follow the proof of [GaP, thm. 1.4].

\section{Lemma 4.1.}

Let $T$ be a smooth projective variety. Let $\mathcal{L}$ be a line bundle on $T$ such that for some $n$ the bundle $\mathcal{L}^{\otimes n}$ gives a birational morphism onto a normal variety and such that the natural map

$$
S^{m} H^{0}(\mathcal{L}) \longrightarrow H^{0}\left(\mathcal{L}^{\otimes m}\right)
$$

is surjective for $m \geq 1$. Then $\mathcal{L}$ gives a birational morphism onto a projectively normal variety.

of lemma. The problem is to show the normality of the image. Consider the following commutative diagram:

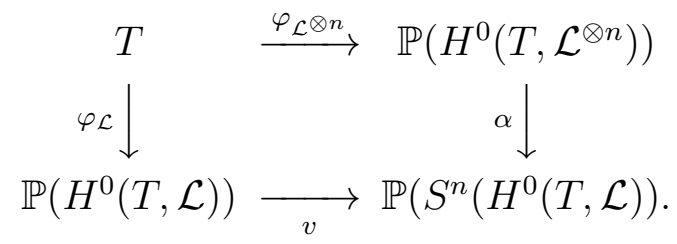

From the assumptions the image of $\alpha \circ \varphi_{\mathcal{L} \otimes n}$ is normal. It remains to observe that $v$ is the Veronese embedding.

We apply the above lemma to $\mathcal{L}=\mathcal{O}_{X}(G)$. By thm. 2.2 there is an $n$ such that $\mathcal{O}_{X}(n G)$ gives a morphism with normal image. Thus, to prove that the assumption of the lemma holds it is enough to show that

$$
H^{0}(n G) \otimes H^{0}(G) \longrightarrow H^{0}((n+1) G)
$$

is surjective for $n \geq 1$. Consider the following commutative diagram:

$$
\begin{aligned}
& H^{0}(n G) \otimes H^{0}\left(\mathcal{O}_{X}\right) \hookrightarrow H^{0}(n G) \otimes H^{0}(G) \rightarrow H^{0}(n G) \otimes H^{0}\left(\left.G\right|_{G}\right) \\
& H^{0}(n G) \quad \hookrightarrow \quad H^{0}((n+1) G) \quad \rightarrow \quad H^{0}\left(\left.(n+1) G\right|_{G}\right) .
\end{aligned}
$$

To show the surjectivity of the middle vertical map it is enough to show the surjectivity of the right-hand-side vertical map as the surjectivity of the left-hand-side map is clear. Since $\left.\mathcal{O}_{X}(G)\right|_{G}=K_{G}$ and the restriction map $H^{0}\left(\mathcal{O}_{X}(n G)\right) \longrightarrow H^{0}\left(n K_{G}\right)$ is surjective, it is enough to prove that

$$
H^{0}\left(n K_{G}\right) \otimes H^{0}\left(K_{G}\right) \longrightarrow H^{0}\left((n+1) K_{G}\right)
$$


is surjective for all $n \geq 1$. This is clear from the fact that $G^{\prime} \subset F \subset$ $\mathbb{P}^{r+1}$ is projectively normal for $r \geq 3$ and it is the canonical model of $G$.

In the case $r=1$ we have $h^{0}\left(\mathcal{O}_{X}(G)\right)=4$. Since $\left(H^{*}+D\right)^{3}=3$, we conclude that $\operatorname{deg} \varphi_{\left|H^{*}+D\right|}=3$.

If $r=2$, then $h^{0}\left(\mathcal{O}_{X}(G)\right)=h^{0}\left(\mathcal{O}_{F}(H)\right)+1=5$ and $\left(H^{*}+D\right)^{3}=6$. Since $\operatorname{deg}\left(\operatorname{im} \varphi_{\left|H^{*}+D\right|}\right) \cdot \operatorname{deg} \varphi_{\left|H^{*}+D\right|}=6$, to prove that $\varphi_{\left|H^{*}+D\right|}$ is $2: 1$ it is enough to observe that $\left.G\right|_{G}$, defines a $2: 1$ morphism onto $X_{3} \subset \mathbb{P}^{3}$.

In order to describe more precisely the images of the obtained primitive contractions we compute $h^{0}\left(\mathcal{O}_{X}(n G)\right)$. From the following exact sequences

$$
\begin{gathered}
0 \longrightarrow \mathcal{O}_{X}((n-1) G) \longrightarrow \mathcal{O}_{X}(n G) \longrightarrow N_{n G \mid X} \longrightarrow 0 \\
0 \longrightarrow \mathcal{O}_{F}((n-3) H) \longrightarrow \mathcal{O}_{F}(n H) \longrightarrow \mathcal{O}_{G}(n H) \longrightarrow 0
\end{gathered}
$$

we obtain as in the proof before

$h^{o}\left(\mathcal{O}_{X}(n G)\right)=h^{o}\left(\mathcal{O}_{X}((n-1) G)+h^{o}\left(\mathcal{O}_{F}(n H)\right)-h^{o}\left(\mathcal{O}_{F}((n-3) H)\right)\right.$.

Using the Riemann-Roch theorem, we have

$$
\begin{gathered}
\chi\left(\mathcal{O}_{F}(n H)\right)=\frac{1}{12} n H(n H+2 H)(2 n H+2 H)+\frac{1}{24} c_{1} c_{2}+\frac{1}{12} n H c_{2}= \\
=\frac{1}{6} r n(n+1)(n+2)+n+1 .
\end{gathered}
$$

By the Kodaira vanishing theorem, this implies that for $n \geq 1$

$$
h^{o}\left(\mathcal{O}_{X}(n G)\right)=\frac{r}{2} n^{3}+\left(\frac{r}{2}+3\right) n .
$$

The graded ring $\oplus_{n=0}^{\infty} H^{0}\left(\mathcal{O}_{X}(n m G)\right)$ is the coordinate ring of the image of $\varphi_{|m G|}$ for $m>>0$. Hence the scheme $\operatorname{Proj}\left(\oplus_{n=0}^{\infty} H^{0}\left(\mathcal{O}_{X}(n G)\right)\right)$ is isomorphic to the Calabi-Yau threefold obtained after the primitive contraction. The Hilbert series of $\oplus_{n=0}^{\infty} H^{0}\left(\mathcal{O}_{X}(n G)\right)$ is the following

$$
P(t)=\frac{t^{4}+(r-1) t^{3}+r t^{2}+(r-1) t+1}{(1-t)^{4}} .
$$

For $r=1$, we obtain

$$
P(t)=\frac{\left(1-t^{6}\right)}{(1-t)^{4}\left(1-t^{2}\right)} .
$$

This is the Hilbert series of a sextic in $\mathbb{P}(1,1,1,1,2)$ (see [R5]). We claim that $\oplus_{n=0}^{\infty} H^{0}\left(\mathcal{O}_{X}(n G)\right)$ is in fact isomorphic to the graded ring of such a variety. Let us choose generators $x, y, z, t \in H^{0}\left(\mathcal{O}_{X}(G)\right)$. Computing the dimension of $H^{0}\left(\mathcal{O}_{X}(2 G)\right)$ we see that we need an additional 
generator of degree 2. It is enough to prove now that $\varphi_{|2 G|}$ gives a primitive contraction onto a projectively normal variety in $\mathbb{P}\left(H^{0}\left(\mathcal{O}_{X}(2 G)\right)\right)$. To show this, we can follow the proof of the case $i \geq 3$ of thm. 4.1, knowing that $\left.2 G\right|_{G}$ gives an isomorphism onto a projectively normal surface in $\mathbb{P}\left(H^{0}\left(\mathcal{O}_{G}\left(2 K_{G}\right)\right)\right)$.

For $r=2$,

$$
P(t)=\frac{\left(1-t^{3}\right)\left(1-t^{4}\right)}{(1-t)^{5}\left(1-t^{2}\right)} .
$$

This gives the Hilbert series of a complete intersection of a quadric and a cubic in $\mathbb{P}(1,1,1,1,1,2)$. We can prove as before that the graded ring $\oplus_{n=0}^{\infty} H^{0}\left(\mathcal{O}_{X}(n G)\right)$ is in fact isomorphic to the graded ring of such a variety.

For $r=3$

$$
P(t)=\frac{\left(1-t^{3}\right)\left(1-t^{3}\right)}{(1-t)^{6}} .
$$

This proves in fact that the image of $\varphi_{|G|}$ is the intersection of two cubics in $\mathbb{P}^{5}$. Indeed, from $h^{0}\left(\mathcal{O}_{X}(3 G)\right)=54, h^{0}\left(\mathcal{O}_{X}(2 G)\right)=21$, and from the fact that the image of $\varphi_{|G|}$ is not contained in a quadric we conclude that it is contained in two cubics without common component. It remains to observe that the image is of degree 9.

For $r=4$,

$$
P(t)=\frac{\left(1-t^{2}\right)\left(1-t^{2}\right)\left(1-t^{3}\right)}{(1-t)^{7}},
$$

we can prove as before that the image of $\varphi_{|G|}$ is a complete intersection of two quadrics and a cubic in $\mathbb{P}^{6}$.

For $r=5$,

$$
P(t)=\frac{\left(-t^{5}+5 t^{3}-5 t^{2}+1\right)\left(1-t^{3}\right)}{(1-t)^{8}} .
$$

This gives a Hilbert series of the intersection in $\mathbb{P}^{7}$ of a cubic and a linear section of the Grassmannian $G(2,5)$ in its Plücker embedding. We shall sketch the proof that the image $\tilde{X}$ of $\varphi_{|G|}$ is such an intersection. The image $\varphi_{|G|}(G)$ is canonically embedded in a hyperplane section $H^{\prime} \simeq \mathbb{P}^{6}$. It follows that the intersection of all quadrics $Q_{G} \subset \mathbb{P}^{6}$ containing $\varphi_{|G|}(G)$ is a smooth linear section of the Grassmannian $G(2,5)$ in its Plücker embedding ( $G^{\prime}$ is such a section). Since $H^{0}\left(\mathcal{I}_{\tilde{X}}(1)\right)=H^{1}\left(\mathcal{I}_{\tilde{X}}(1)\right)=0$, the natural map

$$
H^{0}\left(\mathcal{I}_{\tilde{X}}(2)\right) \rightarrow H^{0}\left(\mathcal{I}_{\tilde{X}} \otimes \mathcal{O}_{H^{\prime}}(2)\right)
$$

is an isomorphism. Denote by $Q$ the intersection of all quadrics containing $\tilde{X}$. We see that $\tilde{X}$ is the intersection of $Q$ with a cubic. We 
shall show that $Q$ is a 4 dimensional Gorenstein irreducible linear section of $G(2,5)$. To prove this, it is enough to show that the fourfold $Q$ is irreducible, Gorenstein, subcanonical, and arithmetically Gorenstein.

Indeed, by the result of Walter [Wa] we obtain that $Q$ is defined by Pfaffians. From the fact that $Q$ is arithmetically Gorenstein (i.e. $\oplus_{j} H^{1}\left(I_{Q}(j)\right)=0$ and $H^{i}\left(\mathcal{O}_{Q}(r)\right)=0$ for all $r$ and $1 \leq i \leq 3$ ) we moreover, obtain from [Wa] that there is a resolution

$$
0 \rightarrow \mathcal{O}_{\mathbb{P}^{7}}(-t) \rightarrow \mathcal{E}^{\vee}(-t) \rightarrow \mathcal{E} \rightarrow \mathcal{O}_{\mathbb{P}^{7}} \rightarrow \mathcal{O}_{Q}
$$

where $t$ is an integer and $\mathcal{E}=\bigoplus_{i=1}^{2 p+1} \mathcal{O}_{\mathbb{P}^{7}}\left(a_{i}\right)$ such that $a_{i}$ are uniquely determined. Since a linear section of $G(2,5)$ is arithmetically Gorenstein and restricts to the hyperplane section as $Q$, the fourfold $Q$ is isomorphic to the linear section of the Grassmannian $G(2,5)$ in its Plücker embedding.

It remains to prove that $Q$ is irreducible, Gorenstein, subcanonical, and arithmetically Gorenstein. Since the space of quadrics containing $\tilde{X}$ is 5-dimensional, each component of $Q$ is at least one-dimensional. Moreover, we know that some hyperplane section of $Q$ is irreducible. Together these imply that $Q$ is irreducible.

We show now that $Q$ is normal and Gorenstein. Since $\tilde{X} \subset Q$ is a Cartier divisor and $\tilde{X}$ is Gorenstein, we conclude that $Q$ is Gorenstein at points of $\tilde{X}$. Next, if $P \in Q-\tilde{X}$, then the generic codimension 2 linear section through $P$ cuts $Q$ along an irreducible surface $S$ of degree 5 in $\mathbb{P}^{5}$.

Claim The surface $S$ is either a cone over a smooth elliptic curve or a Gorenstein del Pezzo surface.

Indeed, from Nagata's $\mathrm{Na}$ classification of surfaces of degree 5 in $\mathbb{P}^{5}$, it is enough to prove that $S$ is neither a projection of a rational normal scroll nor a projection of a cone over a rational normal curve. This follows from the fact that $S$ has a hyperplane section (corresponding to the intersection with $H^{\prime}$ ) which is a smooth elliptic curve but each hyperplane section of a rational normal scroll or a cone over a rational normal curve is rational. The claim follows.

We conclude that $Q$ is normal and Gorenstein. To see that $Q$ is subcanonical, observe that $Q_{G}$ is subcanonical and hence the generic hyperplane section is subcanonical. From the adjunction formula, $K_{Q_{G}}=$ $K_{Q}+\left.Q_{G}\right|_{Q_{G}}$, so $\left.K_{Q}\right|_{Q_{G}}=\mathcal{O}_{Q_{G}}(-2)$. We use now the Lefschetz hyperplane theorem from [RS] to see that there is a unique Weil divisor on $Q$ that restricts to $Q_{G}$ in such a way. It follows that $K_{Q}=\mathcal{O}_{Q}(-3)$, hence $Q$ is subcanonical. 
It remain to prove that $Q$ is arithmetically Gorenstein. From the exact sequence

$$
0 \longrightarrow \mathcal{I}_{Q}(j-1) \longrightarrow \mathcal{I}_{Q}(j) \longrightarrow \mathcal{I}_{Q_{G}}(j) \longrightarrow 0
$$

and the fact that $Q_{G}$ is projectively normal (i.e. $H^{1}\left(\mathcal{I}_{Q_{G}}(j)\right)=0$ ) we obtain that

$$
H^{1}\left(\mathcal{I}_{Q}(j-1)\right) \longrightarrow H^{1}\left(\mathcal{I}_{Q}(j)\right)
$$

is surjective hence $H^{1}\left(\mathcal{I}_{Q}(j)\right)=H^{1}\left(\mathcal{I}_{Q}\right)=0$.

Similarly, from

$$
0 \longrightarrow \mathcal{O}_{Q}(j-1) \longrightarrow \mathcal{O}_{Q}(j) \longrightarrow \mathcal{O}_{Q_{G}}(j) \longrightarrow 0
$$

we obtain that

$$
H^{i}\left(\mathcal{O}_{Q}(j-1)\right) \longrightarrow H^{i}\left(\mathcal{O}_{Q}(j)\right)
$$

is surjective for $1 \leq i \leq 3$. From the Serre duality $H^{i}\left(\mathcal{O}_{Q}\right)=H^{4-i}\left(\mathcal{O}_{Q}(-3)\right)=$ 0 . Thus $Q$ is arithmetically Gorenstein. We have proved the following theorem.

\section{Theorem 4.2.}

For $r=1$, the morphism $\varphi_{|3 G|}$ is a primitive contraction. If $r=2$, then $\varphi_{|2 G|}$ is a primitive contraction, for $r=3,4,5$ the primitive contraction is given by $\varphi_{|G|}$. Moreover,

- if $r=1$, then $\varphi_{|3 G|}(X)$ is isomorphic to a sextic in $\mathbb{P}(1,1,1,1,2)$,

- if $r=2$, then $\varphi_{|2 G|}(X)$ is isomorphic to a complete intersection of a cubic and a quartic in $\mathbb{P}(1,1,1,1,1,2)$,

- if $r=3$, then $\varphi_{|G|}(X)$ is isomorphic to a complete intersection of two cubics in $\mathbb{P}^{5}$,

- if $r=4$, then $\varphi_{|G|}(X)$ is isomorphic to a complete intersection of two quadrics and a cubic in $\mathbb{P}^{6}$,

- if $r=5$ then $\varphi_{|G|}(X)$ is isomorphic to a complete intersection of a cubic and a codimension 2 linear section of the Grassmannian $G(2,5)$ embedded by the Plücker embedding in $\mathbb{P}^{9}$.

Remark 4.1. From the geometric descriptions contained in the previous theorem we can compute the differences between the Euler characteristics $\chi\left(\mathcal{Y}_{t}\right)-\chi(X)$ (cf. Remark 3.4). Since the values of $\chi\left(\mathcal{Y}_{t}\right)$ are known, it is enough to find $\chi(X)$. We do this using the commutative diagram from [C1]

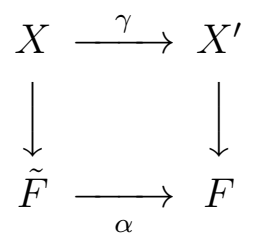


where $\alpha$ and $\gamma$ are blowings up of $F$ and $X^{\prime}$ along $D^{\prime} \cap G^{\prime}$ and its strict transform to $X^{\prime}$, respectively. We need also the additivity of the Euler characteristic

$$
\begin{gathered}
\chi(\tilde{F})=\chi(F)+\chi(G \cap D) \\
\chi(X)=2 \chi(\tilde{F})-\chi\left(D^{\prime}\right)-\chi\left(G^{\prime}\right) .
\end{gathered}
$$

4.2. Construction 2. Let $F_{1}$ be a Fano manifold with Picard number 1 and Gorenstein index greater than 2. From tables in chapter 12 of [IP] we have $F_{1}=\mathbb{P}^{3}$ or $F_{1}$ is a quadric in $\mathbb{P}^{4}$.

- First, let $F_{1}=\mathbb{P}^{3}$. In this case $\left|-K_{\mathbb{P}^{3}}\right|=|-4 H|$ where $H$ is a hyperplane in $\mathbb{P}^{3}$. Let us denote by $D_{i}^{\prime}$, for $i=1,2,3$ generic (in particular smooth) elements of $|i H|$. Then

- $D_{1}^{\prime}=\mathbb{P}^{2}$,

- $D_{2}^{\prime}=\mathbb{P}^{1} \times \mathbb{P}^{1}$,

- $D_{3}^{\prime}$ is the blowing up of $\mathbb{P}^{2}$ in 6 points.

Let us moreover, choose for $i=1,2,3$ generic elements $G_{i}^{\prime} \in|(8-i) H|$.

\section{Lemma 4.3.}

The normal double covers $X_{i}^{\prime}$ of $\mathbb{P}^{3}$ branched along $G_{i}^{\prime}+D_{i}^{\prime}$ are Calabi$Y a u$ manifolds with transversal $A_{1}$ singularities along the strict transforms of $G_{i}^{\prime} \cap D_{i}^{\prime}$.

In the same way as in construction 1 , we resolve $\gamma: X_{i} \rightarrow X_{i}^{\prime}$ and obtain Calabi-Yau manifolds with $\rho\left(X_{i}\right)=2$. As before, let $D_{i}, G_{i}$ be the strict transforms of $D_{i}^{\prime}$ and $G_{i}^{\prime}$ to $X_{i}$. Observe that for each divisor $V \in \operatorname{Pic}\left(X_{i}\right)$, the restriction $\left.V\right|_{D_{i}}$ is a multiple of $K_{D_{i}}$. From thm. 2.2, there exists a type II primitive contraction that contracts $D_{i}$. To be more precise

\section{Theorem 4.3.}

The linear system $\left|G_{i}\right|$ is base-point-free and the corresponding morphism is a primitive contraction. Moreover,

- the image of $\varphi_{\left|G_{1}\right|}$ is a threefold of degree 63 in $\mathbb{P}^{20}$,

- the image of $\varphi_{\left|G_{2}\right|}$ is a threefold of degree 24 in $\mathbb{P}^{10}$, which is an intersection of a cone over the Veronese embedding of $\mathbb{P}^{3}$ in $\mathbb{P}^{9}$ with a generic sextic passing through the vertex,

- the image of $\varphi_{\left|G_{3}\right|}$ is a quintic in $\mathbb{P}^{4}$.

Proof. Observe that $G_{i} \in\left|E+\frac{8-i}{i} D_{i}\right|$. From the adjunction formula

$$
\left.D_{i}\right|_{D_{i}}=K_{D_{i}},\left.\quad E\right|_{D_{i}}=-\frac{8-i}{i} K_{D_{i}} .
$$

We compute as before the Hilbert series of $\oplus_{n=0}^{\infty} H^{0}\left(\mathcal{O}_{X}\left(n G_{i}\right)\right)$ and obtain: 
- If $i=3$,

- If $i=2$,

$$
P(t)=\frac{\left(1-t^{5}\right)}{(1-t)^{5}}
$$

$$
P(t)=\frac{\left(1-t^{3}\right)\left(1+6 t+t^{2}\right)}{(1-t)^{5}} .
$$

This is the Hilbert series of the double Veronese embedding of a sextic in $P(1,1,1,1,2)$.

- If $i=1$,

$$
P(t)=\frac{t^{4}+17 t^{3}+27 t^{2}+17 t+1}{(1-t)^{4}} .
$$

The proof now follows as in example 4.1.

Remark 4.2. Denote by $\mathcal{Y}_{t}$ some smooth Calabi-Yau manifold from the smoothing family of the images $Y_{i}$ of primitive contractions constructed in the previous theorem. We can compute Euler characteristics $\chi\left(\mathcal{Y}_{t}\right)$ without using thm. 4.3. To obtain $\chi\left(X_{i}\right)$ we follow the method described in remark 4.1. The computation is illustrated in the following table.

\begin{tabular}{|c|c|c|c|c|c|c|c|}
\hline$i$ & $D_{i}^{\prime}$ & $G_{i}^{\prime}$ & $\chi\left(D_{i}^{\prime}\right)$ & $\chi\left(G_{i}^{\prime}\right)$ & $\chi\left(G_{i}^{\prime} \cap D_{i}^{\prime}\right)$ & $\chi\left(X_{i}\right)$ & $\chi\left(\mathcal{Y}_{t}\right)$ \\
\hline 1 & $\mathbb{P}^{2}$ & septic & 3 & 189 & -28 & -240 & - \\
\hline 2 & $\mathbb{P}^{1} \times \mathbb{P}^{1}$ & sextic & 4 & 128 & -48 & -200 & -204 \\
\hline 3 & cubic & quintic & 9 & 55 & -60 & -176 & -200 \\
\hline
\end{tabular}

We find values of $\chi\left(\mathcal{Y}_{t}\right)$ using the results from section 3 .

- In the case where $F_{1}$ is a quadric in $\mathbb{P}^{4}$ we have $\left|-K_{F}\right|=|3 H|$, where $H \subset \mathbb{P}^{4}$ is a hyperplane section. Choose del Pezzo surfaces $D_{4}^{\prime} \in|2 H|, D_{5}^{\prime} \in|H|$ (here $D_{4}^{\prime}$ is of degree 4 and $D_{5}^{\prime}$ is isomorphic to $\left.\mathbb{P}^{1} \times \mathbb{P}^{1}\right)$ and generic elements $G_{5}^{\prime} \in|5 H|, G_{4}^{\prime} \in|4 H|$. We construct analogously as before Calabi-Yau threefolds $X_{4}$ and $X_{5}$ with Picard group of rank 2 , and divisors $G_{i}, D_{i} \subset X_{i}$.

Remark 4.3. Using the results from section 3 we see that the threefold $\varphi_{\left|G_{5}\right|}\left(X_{5}\right)$ can be smoothed and that the Hodge numbers of this smoothing are $h^{1,1}=1$ and $h^{1,2}=101$. This lead us to the following theorem.

\section{Theorem 4.4.}

The linear system $\left|G_{i}\right|$ is base-point-free and $\varphi_{\left|G_{i}\right|}$ is a primitive contraction contracting $D_{i}$. 
- The image of $\varphi_{\left|G_{4}\right|}$ is an intersection of a quadric and a quartic in $\mathbb{P}^{5}$.

- The image of $\varphi_{\left|G_{5}\right|}$ is a threefold of degree 40 in $\mathbb{P}^{14}$ that is a degeneration of a family of quintics embedded by the Veronese embedding in $\mathbb{P}^{14}$.

Proof. The proof is similar to the proof of thm. 4.2.

Remark 4.4. We cannot make analogous constructions for other Fano threefolds. It follows from the tables 12.3-12.6 in [IP that all remaining Fano threefolds have a fibration cutting non-canonically the embedded del Pezzo surface.

\section{Construction using Complete intersection}

In this subsection we show another way to construct primitive contractions. The Calabi-Yau threefold that contains the del Pezzo surface is obtained as a resolution of a nodal complete intersection of hypersurfaces that contains the del Pezzo surface in its anti-canonical embedding.

5.1. Construction 3. Let $D^{\prime} \subset \mathbb{P}^{4}$ be an anti-canonically embedded smooth del Pezzo surface of degree 4 . The surface $D^{\prime}$ is a complete intersection of two quadrics $f_{1}, f_{2}$.

Consider the quintic $p=f_{1} g_{1}+f_{2} g_{2}$, where $g_{1}, g_{2}$ are generic cubics. Then $X_{1}=\{p=0\}$ is a Calabi-Yau manifold with 36 nodes at the points where $f_{1}=g_{1}=f_{2}=g_{2}=0$ (taking $f_{1}, g_{1}, f_{2}, g_{2}$ as analytic coordinates near a singular point, $p=0$ is an equation of an ordinary double point).

The smooth surface $S^{\prime}=\left\{g_{1}=f_{2}=0\right\}$ pas through all nodes of $X^{\prime}$. The blowing up of $S^{\prime} \subset X^{\prime}$ is a small resolution of $X^{\prime}$.

Remark 5.1. Since $D^{\prime} \subset X^{\prime}$ is a smooth Weil divisor passing through the nodes, blowing up $D^{\prime}$ produces a small resolution. We can obtain $X$ by flopping the 36 exceptional lines.

\section{Lemma 5.1.}

The Rank of the Picard group of $X$ is 2.

Proof. Since $X$ is a small resolution of a nodal threefold, it is enough to show (see [C1, thm. 2]) that the defect $\delta$ of $X^{\prime}$ is 1 . We know that it is greater than 0 since $D^{\prime}$ is a Weil divisor.

Recall that the defect of $X^{\prime}$ is equal to

$$
h^{0}\left(\mathcal{O}_{\mathbb{P}^{4}}(5) \otimes \mathcal{I}_{S^{\prime}}\right)-h^{0}\left(\mathcal{O}_{\mathbb{P}^{4}}(5)\right)+\mu,
$$


where $\mu$ is the number of nodes and $\mathcal{I}_{S^{\prime}} \subset \mathcal{O}_{\mathbb{P}^{4}}$ is the ideal sheaf of these nodes. Since $\mathcal{I}_{S^{\prime}}$ is a complete intersection of two quadrics and two cubics, Hilbert polynomial computations yield $\delta=1$.

Let $D \subset X$ be the strict transform of $D^{\prime}$ and $H$ the strict transform of the hyperplane section to $X$. Since $S^{\prime}$ is transversal to $D^{\prime}$ we see that $D^{\prime}$ is isomorphic to $D$. The surface $D \subset X^{\prime}$ is a del Pezzo surface such that $\left.D\right|_{D}=K_{D}$ (by the adjunction formula) and $\left.H\right|_{D}=-K_{D}$ (because $D^{\prime}$ is anti-canonically embedded). Thus, $D$ can be contracted in a primitive way.

\section{Theorem 5.1.}

The linear system $|D+H|$ is base-point-free. Moreover, $\varphi_{|D+H|}$ is a primitive contraction with exceptional locus $D^{\prime}$. Its image is a complete intersection of two cubics in $\mathbb{P}^{5}$.

Proof. Let $S \subset X^{\prime}$ be the strict transform of $S^{\prime}$ and $G^{\prime}=\left\{f_{3}=g_{3}=0\right\}$. Observe that $|2 H|=|S+D|$, as $f_{2}$ cuts $X_{1}$ along $S$ and $D$. Analogously $|3 H|=|G+S|$, so $G \in|H+D|$. Since $G \cap D=\emptyset$ we conclude that $|D+H|$ is base-point-free. Moreover, $|D+H|$ gives a birational morphism. This follows from the fact that $|H|$ separates points from $X \backslash D$ and that

$$
(D+H)^{3}=K_{D}^{2}+3 K_{D}^{2}-3 K_{D}^{2}+H^{3}=9 .
$$

To compute the Hilbert series of $\oplus_{n=0}^{\infty} H^{0}\left(\mathcal{O}_{X}(n G)\right)$ we find $h^{0}\left(\mathcal{O}_{G^{\prime}}\left(K_{G^{\prime}}\right)\right)=$ $h^{0}\left(\mathcal{O}_{G^{\prime}}(1)\right)$. Now, since $G^{\prime} \subset \mathbb{P}^{4}$ is projectively normal, we conclude as in the proof of thm. 4.2 .

Remark 5.2. We can perform an analogous construction for del Pezzo surfaces of degree 3 . Let $D^{\prime} \subset \mathbb{P}^{4}$ be the intersection of a generic cubic $c$ and a hyperplane $l$. We find as before a quintic $X^{\prime}$ with 24 node defined by $q=c g_{1}+l_{2}$, where $g_{1}$ is a generic quadric and $g_{2}$ a generic quartic. We construct as before a Calabi-Yau threefold $X$ with Picard group of rank 2 and divisors $D, H \subset X$. We obtain

Theorem 5.2.

The linear system $|D+H|$ is base-point-free. Moreover, $\varphi_{|D+H|}$ is the primitive contraction and its image is a complete intersection of a quadric and a quartic in $\mathbb{P}^{5}$.

5.2. Construction 4. The Grassmannian $G(2,5)=G \subset \mathbb{P}^{9}$ (and so also its generic linear section) is the zero locus of $4 \times 4$ Pfaffians of a generic skew-symmetric $5 \times 5$ matrix with linear entries.

We fix an anti-canonically embedded del Pezzo surface $D^{\prime} \subset \mathbb{P}^{5}$ of degree 5 defined by Pfaffians $p_{1}, p_{2}, p_{3}, p_{4}, p_{5}$, where each $p_{i}$ is obtained 
by deleting the $i$-th row and column from a skew-symmetric matrix $M$.

\section{Lemma 5.2.}

The intersection of two generic cubics $c_{1}, c_{2}$ from the ideal of $D^{\prime}$ is a Calabi-Yau threefold $X^{\prime} \subset \mathbb{P}^{5}$ with 28 ordinary double points. The blowing up of $D^{\prime} \subset X^{\prime}$ is a small resolution. Flopping the 28 exceptional lines we obtain a Calabi Yau threefold X, with two dimensional Kähler cone, containing a del Pezzo surface of degree 5 in a "primitive" way.

Proof. Denote by $l_{1}, \ldots l_{5}, t_{1}, \ldots, t_{5}$ linear forms such that

$$
\begin{aligned}
& c_{1}=l_{1} p_{1}-l_{2} p_{2}+l_{3} p_{3}-l_{4} p_{4}+l_{5} p_{5}, \\
& c_{2}=t_{1} p_{1}-t_{2} p_{2}+t_{3} p_{3}-t_{4} p_{4}+t_{5} p_{5} .
\end{aligned}
$$

Consider the following $7 \times 7$ skew-symmetric matrix $N$.

$$
N=\left(\begin{array}{cc|ccccc}
0 & * & t_{1} & t_{2} & t_{3} & t_{4} & t_{5} \\
& 0 & l_{1} & l_{2} & l_{3} & l_{4} & l_{5} \\
\hline & & & & & & \\
& & & & & & \\
& & & & & &
\end{array}\right) .
$$

Using the fact that the Pfaffian can be expanded along any row, we see that $c_{1}=P_{1}$ and $c_{2}=P_{2}$, where $P_{i}$ is the Pfaffian obtained by deleting the $i$-th row and column from $N$. To see that the singularities of $X^{\prime}$ are nodes, we compute the jacobian matrix of $\left(c_{1}, c_{2}\right)$. The entries of this matrix define an ideal $J$ with support at the singularities of $X^{\prime}$. Let $I$ be the ideal defined by $p_{1}, \ldots, p_{5}, P_{3}, P_{4}, \ldots, P_{7}$. By simple computation in Singular we prove that $J \subset I$ and that $I$ defines a 0-dimensional scheme of degree 28. Moreover, making computations in finite characteristic we see that $I$ is a radical ideal. It remains to observe that $c_{2}\left(\mathcal{I}_{D^{\prime}} / \mathcal{I}_{D^{\prime}}^{2}(3)\right)=28$ (this argument will be made more precise in $[\mathrm{K}]$ ).

To show that $\rho(X)=2$, we first compute

$$
h^{0}\left(\mathcal{O}_{\mathbb{P}^{5}}(3) \otimes \mathcal{I}\right)-h^{0}\left(\mathcal{O}_{\mathbb{P}^{5}}(3)\right)+28=1 .
$$

Since we know that $I$ is the ideal of the set of nodes we conclude arguing as in the proof of theorem 1 from [C1] that $\rho(X) \leq 2$ (the assumptions are not satisfied, so we obtain only an inequality). Now since $X$ is a small resolution and $X^{\prime}$ is not $\mathbb{Q}$-factorial, $\rho(X) \geq 2$. 
The strict transform $D$ of $D^{\prime}$ on $X$ is a del Pezzo surface that can be contracted in a primitive way. Denote by $H \subset X$ the strict transform of a hyperplane section of $\mathbb{P}^{5}$.

Remark 5.3. Using thm. 3.3 we compute that the Hodge numbers of the smoothing of the threefold obtained after the primitive contraction are $h^{1,1}=1$ and $h^{1,2}=50$. This suggests that the threefold in $\mathbb{P}^{6}$ is defined by the $6 \times 6$ Pfaffian of a generic $7 \times 7$ skew-symmetric matrix.

\section{Theorem 5.3.}

The morphism $\varphi_{|H+D|}$ is a primitive contraction. Moreover, its image is a threefold of degree 14 in $\mathbb{P}^{7}$ defined by the $6 \times 6$ Pfaffians of a $7 \times 7$ skew-symmetric matrix .

Proof. Since the $6 \times 6$ Pfaffians of the matrix $N$ define a projectively normal surface $G^{\prime} \subset X^{\prime} \subset \mathbb{P}^{6}$ such that its strict transform in $X$ is an element of $|H+D|$, the first part follows as in theorem 5.1. To prove that the image is defined by Pfaffians, we use the theorem of Walter Wa] (the image have Gorenstein singularities). We know that this image has degree 14 so we conclude as in [Ton] Section 2.

Remark 5.4. In an analogous way we can construct primitive contractions of del Pezzo surfaces of degrees 3 and 4 . Let $D_{1}^{\prime} \subset \mathbb{P}^{5}$ be the intersection of a cubic with two hyperplanes (resp. $D_{2}^{\prime} \subset \mathbb{P}^{5}$ be the intersection of two quadrics $q_{1}, q_{2}$ with a hyperplane). If we choose two generic cubics $c_{1}, c_{2} \in H^{0}\left(\mathcal{I}_{D_{1}^{\prime}}(3)\right)$ (resp. $\left.c_{1}, c_{2} \in H^{0}\left(\mathcal{I}_{D_{2}^{\prime}}(3)\right)\right)$, then the equations $c_{1}=c_{2}=0$ define a Calabi-Yau threefold $X_{1}^{\prime}$ with 12 (resp. $X_{2}^{\prime}$ with 20) ordinary double points. We construct as before threefolds $X_{i}$ with Picard group of rank 2 and divisors $D_{i}, H_{i} \subset X_{i}$.

Theorem 5.4.

The linear system $\left|D_{i}+H_{i}\right|$ is base-point-free. Moreover, $\varphi_{\left|D_{i}+H_{i}\right|}$ is a primitive contraction and

- if $i=1$, its image is a complete intersection of two quadrics and a cubic in $\mathbb{P}^{6}$,

- if $i=2$, the image is defined in $\mathbb{P}^{6}$ by $4 \times 4$ Pfaffians of a $5 \times 5$ skew-symmetric matrix with one row and one column of quadrics and with the remaining entries being linear forms.

\section{REFERENCES}

[Alt] Altmann, K., The versal deformation of an isolated toric Gorenstein singularity Invent. math. 128 (1997), 443-479.

[A] Artin, M., Algebraisation of formal moduli I, Global Analysis, Univ. Tokyo Press, Tokyo and Princeton Univ.Press, Princeton, NJ, 1969. 
[Bin] Bingener, J., Offenheit der Versalit at in der analytischen Geometrie, Math. Z. 173 (1980), 241-281.

[C1] Cynk, S., Defect of a nodal hypersurface, Manuscripta math. 104 (2001), 325-331.

[C2] Cynk, S., Cyclic coverings of Fano threefolds, Ann. Polon. Math. 80 (2003), 117-124.

[C3] Cynk, S., Cohomologies of a double covering of a nonsingular algebraic 3folds, Math. Z. 240 (2002), no. 4, 731-743.

[Har] Hartshorn, R., Algebraic Geometry, Springer-Verlag (1977).

[Fle] Flenner, H., Ein Kriterium fur die Offenheit der Versalitat, Math. Z. 178, 449-473.

[G1] Gross, M., Deforming Calabi-Yau threefolds, Math. Ann. 308 (1997), 187220.

[G2] Gross, M., Primitive Calabi-Yau Threefolds, J.Differential Geometry 45 (1997), 288-318.

[G3] Gross, M., The Deformation Space of Calabi-Yau n-folds with Canonical Singularities can be Obstructed, in Essays in Mirror Symmetry II.

[GaP] Gallego, F.J., Purnaprajna, B.P., Very ampleness and higher syzygies for Calabi-Yau threefold, Math. Ann. 312 (1998), 133-149.

[GP] Gross, M., Popescu, S., Calabi-Yau Threefolds and Moduli of Abelian Surfaces I, Compositio Math. 127, no. 2 (2001), 169-228.

[GH] Green, B., Hubsch, T., Conecting Moduli Spaces of Calabi-Yau Threefolds, Comm. Math. Phys. (1988), 119, 431-441.

[K] Kapustka, G., Primitive contractions of Calabi-Yau threefolds II, arXiv:0707.2488 1 [math.AG].

[Kl] Kleiman, S., Picard scheme, arXiv:math/0504020v1 [math.AG].

[KL] Kleppe, H., Laksov, D., The algebraic structure and deformation of Pfaffian schemes, J. Algebra 64 (1980), 167-189.

[KM] Kollár, J., and Mori, S., Classification of Three-dimensional Flips, J. Amer. Math. Soc., 5 (1992), 533-703.

[KM1] Kollár, J., and Mori, S., Birational geometry of algebraic varieties, Cambridge University Press 134, 1998.

[IP] Iskovskikh, V.A., Prokhorov, Yu.G., Algebraic Geometry V: Fano varieties Springer 1999 .

[MS] Morrison, D.R., Seiberg, N., Extremal transitions and fivedimensional supersymmetric field theories, Nuclear Phys. B 483 (1997), 229247.

[Na] Nagata, M., On rational surfaces I, Mere. Coll. Sci. Kyoto (A) 33 (1960), 352-370.

[N] Namikawa, Y., On deformations of Calabi-Yau 3-folds with terminal singularities. Topology 33 (1994), no. 3, 429-446.

[N1] Namikawa, Y., Deformation theory of Calabi-Yau threefolds and certain invariants of singularities, J. Alg. Geometry 6 (1997), 753-776.

[NS] Namikawa, Y., Steenbrink, J.H.M., Global smoothing of Calabi-Yau threefolds. Invent. Math. 122 (1995), no. 2, 403-419.

[Ni] Nikulin, V.Diagram method for 3-folds and its application to Kähler cone and Picard number of Calabi-Yau 3-folds. I. Higher dimensional complex varieties: Proc. of Intern. Confer. held in Trento, Italy, June 15-24, (1994). (eds. M. Andreatta, Th. Peternell) de Gruyter, (1996), 261-328. 
[RS] Ravindra, G.V., Srinivas, V., The Grothendieck-Lefschetz theorem for normal projective varieties, J. Algebraic Geom. 15 (2006), 563-590.

[R1] Reid, M., The moduli space of 3-folds with $K=0$ may nevertheless be irreducible, in Hirzebruch Festschrift, Math. Ann. 278 (1987), 329-334.

[R2] Reid, M., Constructing algebraic varieties via commutative algebra, in Proc. of 4th European Congress of Math (Stockholm 2004), European Math Soc. 2005, pp. 655-667.

[R3] Reid, M., Canonical 3-folds, Géometrie Algébraique Angers, (A. Beauville, ed.), Sijtho K+Noordhoof (1980), 273-310.

[R4] Reid, M., Chapters on algebraic surfaces, in Complex algebraic varieties, J. Kollár Ed., IAS/Park City lecture notes series (1993 volume), AMS, 1997, $1-154$.

[R5] Reid, M., Graded rings and varieties in weighted projective space http://www.maths.warwick.ac.uk/ miles/surf/more/grad.pdf.

[YPG] Reid, M., Young person's guide to canonical singularities, in Algebraic Geometry, Bowdoin 1985, ed. S. Bloch, Proc. of Symposia in Pure Math. 46, A.M.S. (1987), vol. 1, 345-414.

[S1] Schlessinger, M., Rigidity of Quotient Singularities, Inv.Math. 14 (1971), $17-26$.

[S2] Schlessinger, M., Functors of Artin rings, Trans. AMS 130 (1968), 208-222.

[Ton] Tonoli, F., Construction of Calabi-Yau 3 -folds in $\mathbb{P}^{6}$, J. Algebraic Geom. 13 (2004), 209-232.

[Wa] Walter, Ch., Pfaffian subschemes, J. Alg. Geometry 5 (1996), 671-704.

[Wi1] Wilson, P.M.H., Calabi-Yau manifolds with large Picard number, Invent. Math. 98 (1989), no. 1, 139-155.

[Wi2] Wilson, P.M.H., The Kähler Cone on Calabi-Yau Threefolds, Invent. Math. 107 (1992), 561-583.

[Wi2'] Wilson, P.M.H., Erratum to The Kähler Cone on Calabi-Yau Threefolds, Invent. Math. 114 (1993), 231-233.

[Wi3] Wilson, P.M.H., Towards birational classification of algebraic varieties, Bull. London Math. Soc. 19 (1987), 1-48.

Grzegorz Kapustka

Jagiellonian University

ul. Reymonta 4

30-059 Kraków

Poland

email:

Grzegorz.Kapustka@im.uj.edu.pl

Michal.Kapustka@im.uj.edu.pl
Michał Kapustka

Jagiellonian University

ul. Reymonta 4

30-059 Kraków

Poland 